\title{
Calcium Requirement and Adaptation in Adult Men
}

The needs of the human body for calcium have been investigated frequently by balance studies in which the minimum requirements to maintain calcium equilibrium have been assessed, for which widely varying estimates have been given. These have varied between 200 and $1000 \mathrm{mg}$. daily as an average, but have not always taken into account the influence of old age and osteoporosis.

Ole J. Malm* has recently published a detailed research into this problem and reviewed critically previous reports dating back to 1905 . He describes his method of selecting his experimental subjects, and the two types of diet which he employed. The subjects chosen were long-term convicts and the two diets used contained exactly 950 and 450 nig. calcium daily.

Fourteen day metabolic periods were studied and exact balances of calcium intake and output were measured.

With certain reservations, the author concludes that there was no statistically significant difference in regard to absorption efficiency and calcium retention in men below and above age 50. Osteoporosis in old age has probably several unrelated causes, but one of these factors which has been little recognized, and is shown in these studies, is the influence of physical exercise on the ability to store calcium in old age. The prevention of old age deniineralization may, as Dr. Malm states, prove to be a question not only of adequate nutrition, but also of healthy degrees of exercise.

A better balance also seemed to occur in the summer months, so that some seasonal rhythm of calcium retention seems to take place; this seasonal variation seemed to be due to variations in faecal calcium, suggesting some alteration in calcium absorption. Inverse relationship between urinary and faecal excretion of calcium was inconstant. Prolonged malnourishment seemed to condition the subjects to a less efficient utilization of calcium, but age alone did not itself appear to be correlated to calcium requirements, or to the output of calcium in the urine; nor was body weight or body surface area.

0. J. Malm (Scand. J. Clin. and Lab. Invest., 1958, 10, Suppl. 36). 